\title{
Research on Blind Boxes Consumers---Taking Pop Mart as an Example
}

\author{
Xiaolin Chen ${ }^{1, *}$ \\ ${ }^{1}$ Harbin No.3 High School, Harbin, Heilongjiang Province, China \\ *Corresponding author. Email:1831489715@qq.com
}

\begin{abstract}
Blind boxes are nowadays new toys both native and abroad. People do not know what is inside before opening it. Recently, there are too many people who would like to spend money on them. In some first-tier cities like Shanghai, Guangzhou, almost every shopping mall has at least one blind boxes department. This paper will analyze the reasons why people would like to spend money on it and customer psychology behind them taking the pop mart as an example. It can be concluded that blind boxes have satisfied consumers' psychology of curiosity, pursuing beauty, nostalgia mood, and the desire of companion
\end{abstract}

Keywords: Blind boxes, consumer psychology, public value of consuming.

\section{INTRODUCTION}

Blind boxes came from Japan firstly, which was derived from some cartoon characters in Japan. The same series of blind box has the same appearance; customers cannot tell which type they buy before opening it. In the native, some blind box brands such as pop mart are much popular among people. Some automatic machines aiming to sell blind boxes appear more on each floor of every shopping mall. As a result, research surveys for approximately 170 people by random to survey the reasons for the psychology of people buying blind boxesand the reasons with the research methodology: questionnaires. After finishing the research, people can have a deep understanding of customers' psychology about buying products

\section{EXPERIMENT}

To research the reasons for attracting customers buying blind boxes, this study chooses 200 people of different ages group as research subjects by random (buying and non-buying).

\subsection{Gender}

Among these people, only 85 people purchase blind boxes. Bu calculating, $62 \%$ are women, and the rest of $38 \%$ of people are men (as is shown in Figure 1). As a result, girls prefer buying blind boxes to boys.

\section{the percentage of customers buying blind boxes}

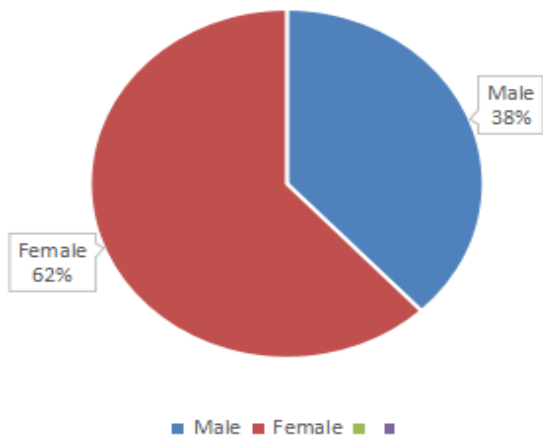

Figure 1 The percentage of customers buying blind boxes 


\subsection{Age}

Besides, Figure 2 shows the percentage of people purchasing blind boxes in different ages group(10-20/ 21-40/41-60). Obviously, blind box toys are loved by most of the young between 10-20, accounting for $85 \%$. However, there are still $12 \%$ of customers in the age group of 21-40. Only 3\% of old people(41-60)choose to buy blind boxes. According to the data above, girls between 10-20 years old usually like to buy blind boxes.

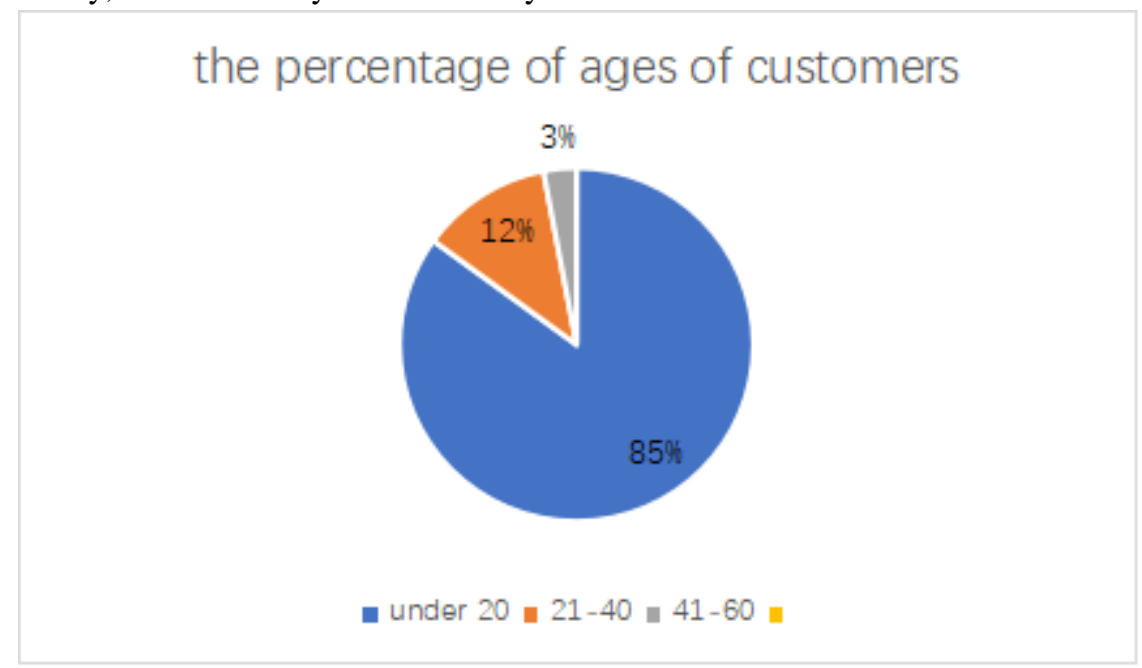

Figure 2 The percentage of ages of customers

\subsection{Reasons for people buying Blind boxes}

reasons for people buying blind boxes. This study have made 100 questionnaires to survey, as is presented in

Moreover, to have a better understanding of the Figure 3.

\section{reasons for buying blind boxes}
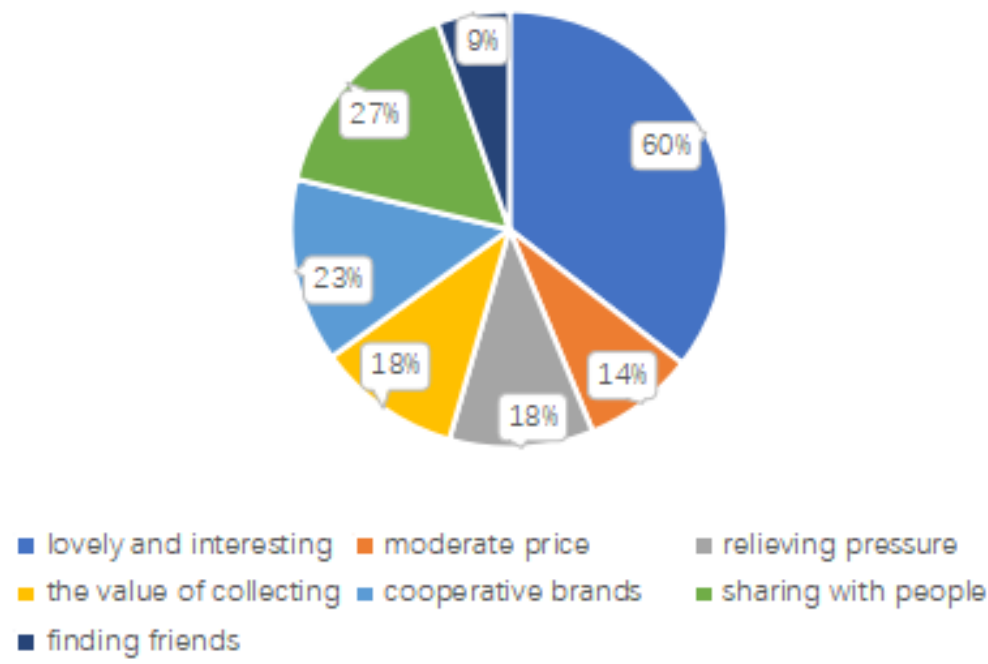

Figure3 Reasons for buying blind boxes

It is really apparent that most people love buying blind boxes because they are lovely and interesting. Nowadays, like some blind boxes brands such as pop mart, which is well-known to the young people. According to the official website, the manager shows different types of blind boxes, such as MOLLY, which is the first member of the whole blind boxes' characters. By surfing the website of MOLLY, people can see many related characters, such as Journey to the West(which is one of the famous fiction in traditional
China), beautiful princesses. MOLLY's designer, Kenny Wang, points out that the characters of MOLLY are from a little young girl wearing a beret hat, which has the same features as MOLLY(big eyes, pouting mouth). After meeting her, Kenny made the first MOLLY in the world. Besides MOLLY, there are also many other characters in the pop mart brand, such as THE MONSTERS, PUCKY, DIMOO and SATYR RORY(all of these toys can be found on the official website). Besides these lovely toys, [2] the gambling feature 
attracts customers. To appeal to people who continue to buy blind boxes, the manager also designs styles of "special edition and hiding edition". Special edition means that this kind of style is scarce of the whole set (less than $1 \%$ ). Some people take the advantage of it to sell the special one at a high price. For example, the original price of a blind box is 59 yuan, but the special edition can achieve 900yuan. The hiding editions (which do not show on the appearance of the box) have the same theory. When people are provided such toys, some of them will be happy because of choosing their favorite style; others will be sad because they seem upset. Such its gambling behaviors mixing mystery and the feeling of surprise, people will purchase them again and again until choosing their favorite style, leading to the sales volume increase, which also represents in Figure 3(accounting to $18 \%$ called the value of collecting). Cooperative brands with blind boxes are also the key to having good sales. Such as pop mart cooperates with different types of brands, like line friends, hello kitty, and gudetama and so on. (23\% showing in the figure). Blind boxes are also popular as a gift sending to people. Nowadays, some young girls, especially 10-20 years old, choose to send blind boxes to their friends as birthday gifts. An interview aims to a boy, who is at Grade 10 said that, "I have sent a blind box to my girlfriend. I will feel happy when she feels great. Some classmates will also exchange blind boxes to gain what they want in the whole set. The last reason, moderate price. For current toys such as LEGO or soft toys, the single price even reaches $100+$. Compared with these toys, blind boxes have a low price. A single box sells at 59-69 yuan. It can not only satisfy the relationship between consumers and characters, but also make the burden on buying them. However, some customers do not buy only one, they will collect all styles in one certain set. Taking PUCKY HOROSCOPE BABIES SERIES as an example, the whole set includes 12 styles and 1 hidden one, which cost 118 dollars in the official shop. Among them, people cannot guarantee every box has different characters inside, so they will buy one set many times, which may spend a lot of money on it totally.

\section{the expense spending on blind boxes}

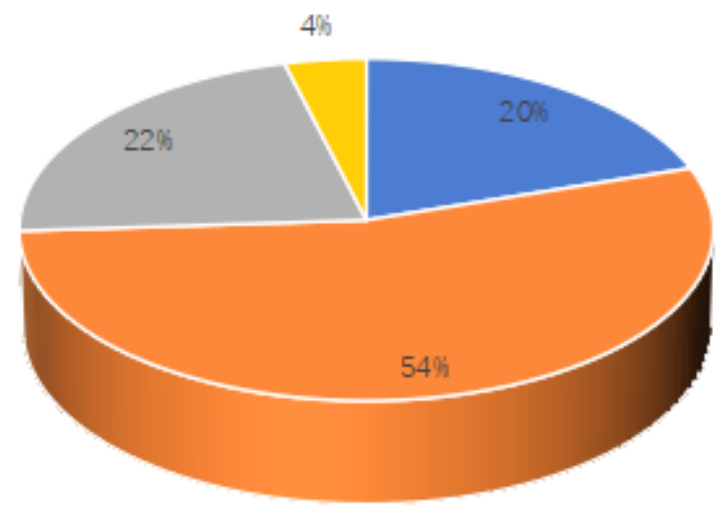

- less than $100 \mid=100-500=500-1000=$ over 1000

Figure 4 The expense spending on blind boxes)

\subsection{Expenses on buying blind boxes}

Figure 4 above shows the percentage of the expense on buying blind boxes totally. Data shows that $54 \%$ of people spend money on blind boxes between 100-500, which accounts for most people. $22 \%$ of people spend 500-1000 yuan on buying blind boxes. All of the figure presents that some customers cannot control themselves from buying blind boxes toys. When they buy a single one, they cannot help themselves buying another style by curiosity. During these years, blind box company like pop mart has won much profit. In 2017, retained profit has reached 7935.3 thousand yuan. Then in 2018, the total operating income has arrived at 161 million yuan, and its retained profit increased to 21098.5 thousand yuan, rising $1405.29 \%$ compared with 2017 . In 2020, pop mart became a listed company.

\section{CONSUMER PSYCHOLOGICAL NEEDS THAT BLIND BOXES CAN MEET}

Due to the fact that, lots of people begin to buy blind boxes for different reasons, there is a new trend formed by blind boxes, which is called 'blind boxes economic'. The consuming psychology behind the blind box needs to be paid attention to. 


\subsection{Pursuing Beauty of everyone}

According to the figure shows above, $60 \%$ of people choose to buy blind boxes because of their lovely and cute appearance without any purpose or any intention. So scientists called this psychology pursuing beauty. [1]Human beings all have an aesthetic value to tell whether the products' appearances are appealing. At the same time, people are full of curiosity about many wonderful people or goods. Such these attracting goods may stimulate customers to buy, however, some of these things are probably useless. According to the mass culture, blind boxes begin to be popular at the beginning of 2018, blind boxes utilize social media and advertisements to announce their products on other social platforms like RED and TikTok[1]. Once people buy certain a kind of blind box and share, more people start to pay attention to such these cute 'babies'. As time goes on, blind boxes gradually are accepted by the public, and these brands can attain more sales. The more sales volume they acquire, the more economic profit they may get. This is also the reason why the blind boxes brand would like to cooperate with different designers to make colorful toys and other similar products, such as pens, bottles and excellent earphones sleeve.

\subsection{Curiosity of Consumers}

Secondly, blind boxes take advantage of people's curiosity to develop their economy. In fact, the feature of the 'blind'. Before opening the box, customers cannot recognize which kind of toy they choose. After buying and opening, customers can realize which style they purchase, common one or hidden one. The pioneers of blind boxes make the usage of the psychology of curiosity and announce by internet, attracting more people to know these toys. People want to learn about new things and situations they have never seen before. For people who never buy blind boxes, they want to know what is inside the small boxes, however for those who are addicted to blind boxes, they might purchase a series of blind boxes, the experience the surprise that choosing their favorite and the sadness when choosing their disliked one force them continuing buying.

\subsection{The nostalgia mood in everyone's heart}

Thirdly, people's nostalgic psychology can help blind box toys to increase their economy. Nostalgic psychology actually represents an experience of emotion, not only being sad or blue when recalling something. Such this psychology come from people's personal experience including watched movies or TV plays before, listened to songs or favorite celebrities. Using pop mart as an example, nowadays the designers of pop mart not only make their own characters of blind boxes such as MOLLY, they but also try to cooperate with their brands from different countries. For example, they design a series of blind boxes about KUNGFU Panda or LINE FRIENDS, BT21 from Korea(such as these familiar brands). People once notice their favorite film characters cooperated with blind boxes; people can't help buying such products. [6]Currently, a famous Chinese film called Detective Chinatown vol.3 is shown all over China. Many young people are fond of these main 'detectives' in this film. As a result, one brand of the blind box designs similar characters to use in their toys. Therefore, people buy this kind of blind boxes because of their favor of film characters.

\subsection{The desire of companion of people}

According to the theory of customers' psychology, it means a phenomenon that consumers modify and control their consuming behaviors under the social environment. With the development of society, people are busier than before. 24-7 routine of working and much competition pressure make them stressed. Gradually, people become lonely and incommunicative. Therefore, they purchase all kind of toys as subjects to help them relieve their pressure and be a friend with these toys. When purchasing and enjoying sharing the toys people get, they will gain a circle of 'blind boxes', which is gathered with like-minded people who have the same hobbies as them. For instance, they can hang out with each other and purchase then sharing them with those friends. According to the theory of Karl Marx and Friedrich Engels, they point out social communication is formed by people's connection, communication and exchange under the environment of production and other social activities. That is how a blind box social community forms.

\section{OTHER INFLUENCE ON OTHER ECONOMY (WE-MEDIA AND TOYS MARKET)}

\subsection{Effects on We-media}

Buying blind boxes, not only can promote the development of the brands of the blind boxes, but also it can also increase the income of other We-media companies. [6]It is really common to notice that there is an increase in the trend of We-media platforms such as RED and TikTok. People edit films and photos, sharing them on their private accounts. Such these people consist of a company and make money with the increasing number amount of playing. Recently, people follow the fashion, recording their vlog of purchasing, opening it. Even, there are people who buy a series of blind box to introduce and attract people coming to see. For example, there 'Youtuber' create topics, sharing their skills of buying hidden or special ones to gain a number of fans. As a result, this is a kind of way to stimulate the chain of products to blind boxes. 


\subsection{Future economy developing in the toys market}

In the future, in about five years, blind boxes will last be an important tool to promote the increase of the economy. According to the figure of the proportion and forecast of the market size of China's toy industry in the global toy industry market from 2017 to 2023. It is clear that, in 2023, the proportion of people buying toys will have reached $23.03 \%$. China toys' industry forces the development of blind boxes. Analyzing the scale of the Chinese toys industry, in 2023, the scale of the industry will increase to 57.46 billion. Among these, there are 9.3 billion involving blind boxes toys. With the developing number of the young especially in the advanced cities, blind boxes will gradually become more popular among people. However, there are also appearing some black market managers who take the advantage of blind boxes to earn money. For example, they will buy lots of series of blind boxes to have hidden or special ones and sell them at a high price on some second-hand platforms. Terribly, some merchants forge the same appearance as some special blind boxes and sell them to earn inappropriate money. If the government wants to keep increasing the sales of blind boxes, they should guarantee the rights of these toys, at the same time, they have to supervise and manage these traders. For the customers, they should purchase blind boxes within their property limits and control the impulse of buying them without addiction.

\section{the developing potential in China's blind boxes market}

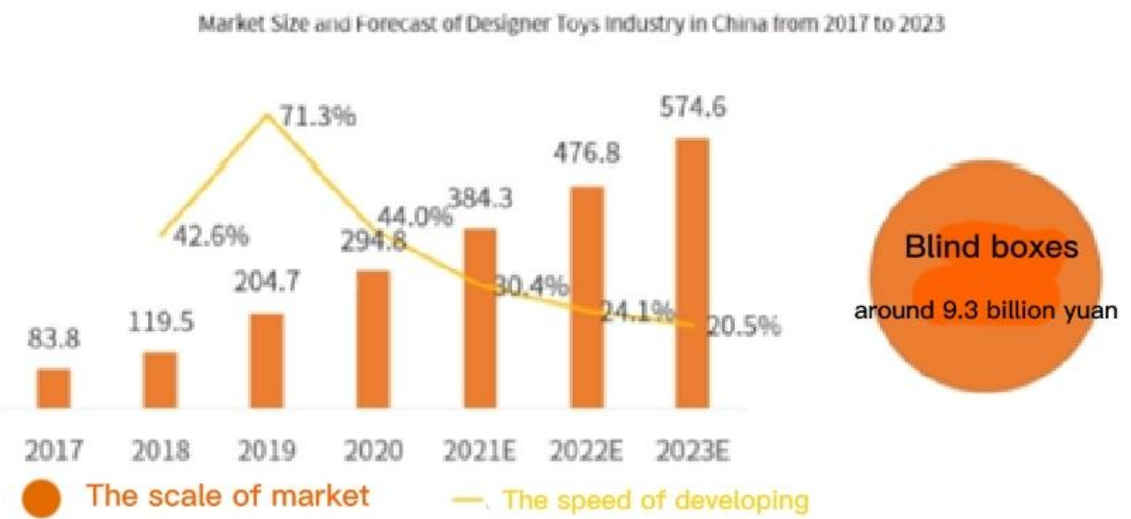

Figure5 The developing potential in China's blind boxes market

\section{Trend2: the driving force of toys' market : blind boxes}

\section{The proportion and forecast of the market size of china's tide play industry in the global tide play market from 2017 to 2002}

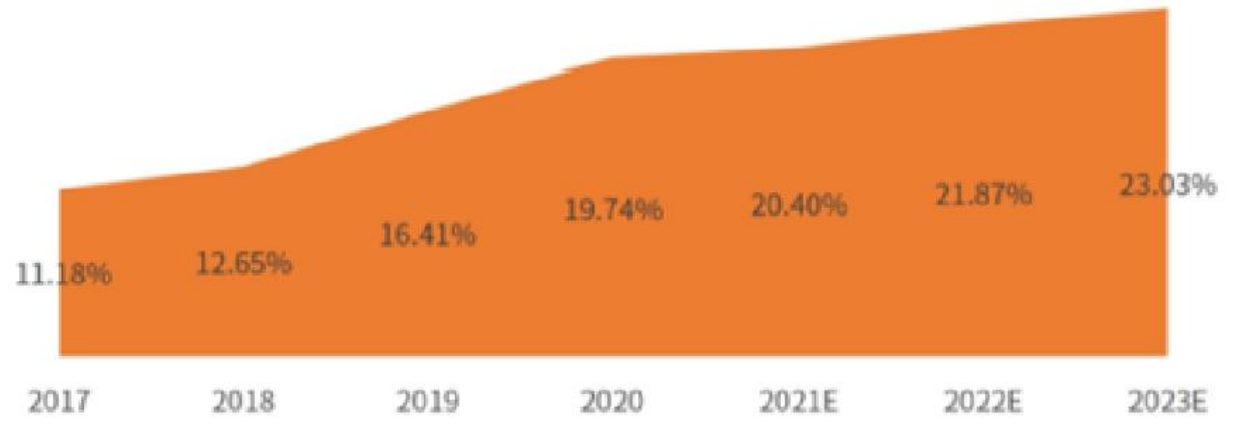

Figure6 The driving force of toys' market: blind boxes

\section{CONCLUSION}

In a fast-paced life, the emerging of the blind boxes completely unexpected. For these consumers of buying blind boxes, most of the girls buy them because of their cute appearance. Cooperating with other famous is still 
the feature of blind boxes. In the future, blind boxes are not only supposed to design different kinds of toys, but also some brands should design more digital facilities, such as earphones with PUCKY, or pencil boxes with MOLLY. Even managers can come up with other kinds of blind boxes besides toys in them. In addition, the department of blind boxes cannot be limited in big cities. To drive the development of the economy in the whole of China, it is better to set up more multiple shops in some small cities.

\section{ACKNOWLEDGMENT}

First, I would like to show my deepest gratitude to my teachers and professors in my high school, who have provided me with valuable guidance in every stage of the writing of this thesis. Further, I would like to thank all my friends and roommates for their encouragement and support. Without all their enlightening instruction and impressive kindness, I could not have completed my thesis.

\section{REFERENCES}

[1] Ruyi Ji, the culture of blind boxes customers' psychology under the vision of internet spreading, Shanghai University institution of news spreading, 2020: 117-119

[2] Alice, the psychology behind the blind boxes, Chinese Glasses technology Magazine,2020/7: 57-61

[3] Yuhuan Lu, the customer psychology and investment research of 'the culture of blind boxes' under the aspect of subculture, Yanbian University, 2020: $17-18$

[4] Zeyuan Zhang, the research of customers' purchasing willing depending on the model of Logistic, Beijing economy and trade College, 2020: 125-131

[5] Guangsi Zhu, what makes blind boxes crazy, The popular Science, 2020: 116-117

[6] Minfang Huang, Jianchang Yuan, the reason analysis of blind boxes fast development, National circulation economics, 2021: 21-23 